\title{
Self-Organizing Map and Relational Perspective Mapping for the Accurate Visualization of High-Dimensional Hyperspectral Data
}

\author{
Wil Gardner ${ }^{1,2,3}$, Ruqaya Maliki ${ }^{1,2}$, Suzanne M. Cutts ${ }^{2}$, Benjamin W. Muir ${ }^{3}$, Davide \\ Ballabio $^{4}$, David A. Winkler ${ }^{2,5,6,7}$ and Paul J. Pigram ${ }^{1 *}$. \\ 1 Centre for Materials and Surface Science and Department of Chemistry and Physics, La Trobe \\ University, Melbourne, Victoria, Australia \\ 2 La Trobe Institute for Molecular Sciences, La Trobe University, Melbourne, Victoria, Australia \\ 3 CSIRO Manufacturing, Clayton, Victoria, Australia \\ 4 Milano Chemometrics and QSAR Research Group, Department of Earth and Environmental Sciences, \\ University of Milano-Bicocca, P.zza della Scienza 1, 20126, Milano, Italy \\ 5 Monash Institute of Pharmaceutical Sciences, Monash University, Parkville, 3052 Australia \\ 6 School of Pharmacy, University of Nottingham, Nottingham NG7 2RD, UK \\ $7 \quad$ CSIRO Data61, Melbourne, Australia \\ * Corresponding Author: p.pigram@latrobe.edu.au
}

\begin{abstract}
This supporting information contains a complete description of the experimental protocol used for the animal study performed for this work, including the methods used for the excision of tumors and subsequent tissue section preparation, as well as spheroid preparation, for time-of-flight secondary ion mass spectrometry (ToF-SIMS) analysis. Also included is the modified relational perspective mapping (RPM) algorithm used in this work, as well as the following figures: Pearson correlation between distance in the SOM-RPM model and in the raw data for different training epochs; schematic showing how the distance correlation metric is calculated; comparison of RGB and CIE $\mathrm{L}^{*} \mathrm{a} \mathrm{b}^{*}$ color schemes; self-organizing maps (SOMs) and corresponding similarity maps colored using the modified RPM algorithm, with and without the topological information provided by the SOM; results from SOM-RPM analysis of ToF-SIMS data described in the main text, using the cosine distance metric for the RPM component of the algorithm; comparison of SOM-RPM, t-SNE and UMAP algorithms, for a spheroid cross section; and mass spectra and weights extracted from regions of interest (ROIs) defined in the main text, as well as corresponding linear discriminant analysis (LDA) results.
\end{abstract}

\section{Contents}

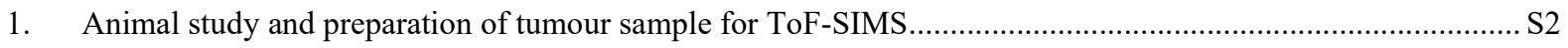

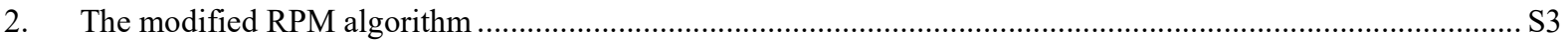

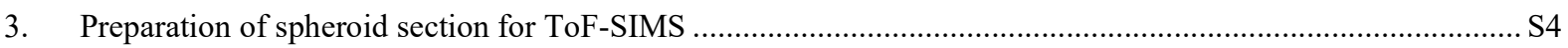

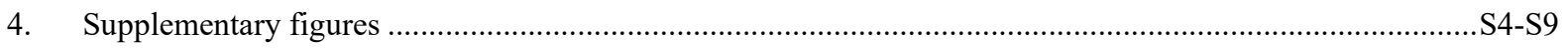

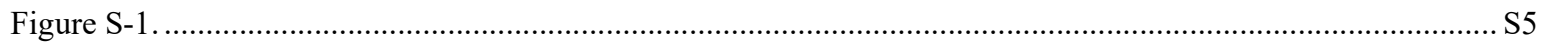

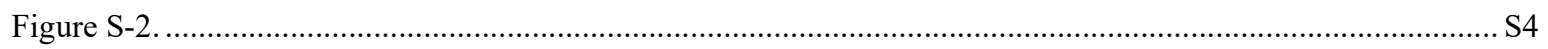

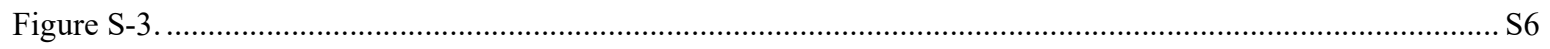

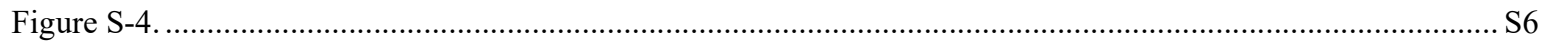

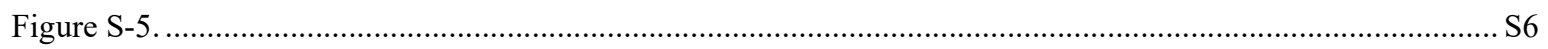

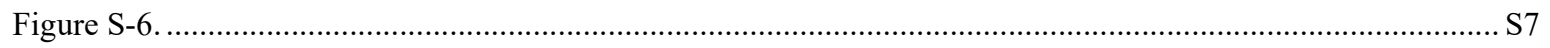

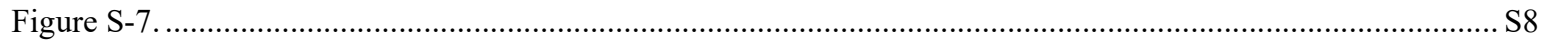

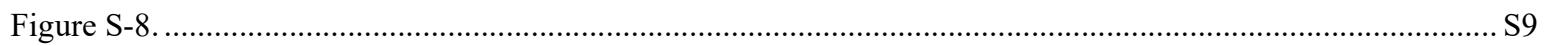

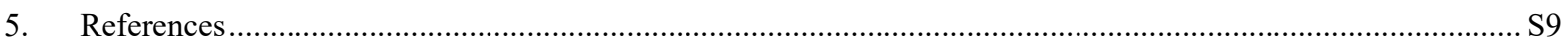




\section{Animal study and preparation of tumour sample for ToF-SIMS}

All animal procedures were carried out at the La Trobe Animal Research and Teaching Facility (LARTF, Melbourne, VIC, Australia) following approval by the La Trobe Animal Ethics Committee, and in accordance with the State legislation and the NHMRC Australian Code for the Care and Use of Animals for Scientific Purposes.

The 4T1.2 mouse triple negative breast cancer (TNBC) cell line was derived in Professor Robin Anderson's laboratory (Olivia Newton-John Cancer Research Institute, Melbourne, VIC, Australia) ${ }^{\mathbf{1}}$ and engineered to express the mCherry and luciferase reporter genes in Associate Professor Belinda Parker's laboratory (La Trobe University, Melbourne, VIC, Australia). ${ }^{2}$ Cells were cultured and maintained in RPMI 1640 medium substituted with $10 \%$ fetal bovine serum and passaged using trypsin-EDTA (ThermoFisher). Cells were verified to be mycoplasma negative by the Victorian Infectious Diseases References Lab when initially frozen-down (Melbourne, VIC, Australia).

A BALB/c mouse was purchased at 8 weeks of age from the Walter and Eliza Hall Institute of Medical Research Institute (Melbourne, VIC, Australia) and allowed one week to acclimatize to the animal facility. The mouse was kept in a $12 \mathrm{~h}$ light-dark cycle and allowed free access to food and water. To generate an orthotopic TNBC tumour, $1 \times 10^{5} 4 \mathrm{~T} 1.2$ cells were re-suspended in PBS and injected into the $4^{\text {th }}$ mammary fat in a $20 \mu \mathrm{l}$ volume on day 0 . Doxil (4 mg/ $\mathrm{kg}$ doxorubicin; Janssen-Cilag, NSW, Australia) was administered intravenously on days 7, 10, 14 and 17; anti-PD-1 (250 $\mu$ g; RPM114, Bioxcell, West Lebanon, NH, USA) was administered intraperitoneally on days 8, 11 and 15. Mouse was ethically euthanized by carbon dioxide asphyxiation on day 17 , five hours after final doxil treatment. Tumor was harvested from the mammary fat pad, placed in PBS, and transported on ice to the Cutt's Laboratory. Tumor was then subsequently embedded in OCT-embedding medium in a beaker containing cooled isopentane settled in a larger beaker of liquid nitrogen. To avoid freezer burn, sample was wrapped in aluminium foil and placed in a sealable plastic bag before storage in $-80^{\circ} \mathrm{C}$.

Prior to cryosectioning, the OCT-embedded sample was transferred to the cryostat and allowed to equilibrate to $-20^{\circ} \mathrm{C}$. Sections (10 $\mu \mathrm{m}$ in thickness) were obtained using a cooled blade, tweezers and paintbrush; the paintbrush was used to hold down the tissue section and prevent it from curling upwards; the tweezers were used to press the ITO-coated glass slide to the tissue section to mount. To avoid further damage by air, the tissue slide was submerged in $95 \%$ ethanol and allowed to air dry before final drying under vacuum. 


\section{The modified RPM algorithm}

The modified RPM algorithm used in the SOM-RPM method is presented below. Refer to the main text and the original RPM algorithm presented by $\mathrm{Li}^{3}$ for more information about the inputs and outputs of the algorithm.






\section{Preparation of spheroid section for ToF-SIMS}

Spheroids were seeded with $66 \mathrm{cl} 4 \mathrm{pBabe}$ and $66 \mathrm{cl} 14 \mathrm{beta} 3$ cell lines in a 96-well round-bottomed plate. Cells were centrifuged for $5 \mathrm{~min}$ at $300 \mathrm{~g}$ then incubated at $37^{\circ} \mathrm{C}$ and $5 \% \mathrm{CO}_{2}$ for 3 days. Aspirated spheroids were transferred to wells containing $10 \%$ formaldehyde then washed with PBS. Cells were cryoprotected with $30 \%$ sucrose solution at $4^{\circ}$ $\mathrm{C}$ overnight, then embedded in OCT and frozen in an isopentane solution cooled with liquid nitrogen. Cryosectioning and subsequent ToF-SIMS analysis were performed similarly to the tumor tissue sections described earlier and in the main text.

\section{Supplementary figures}
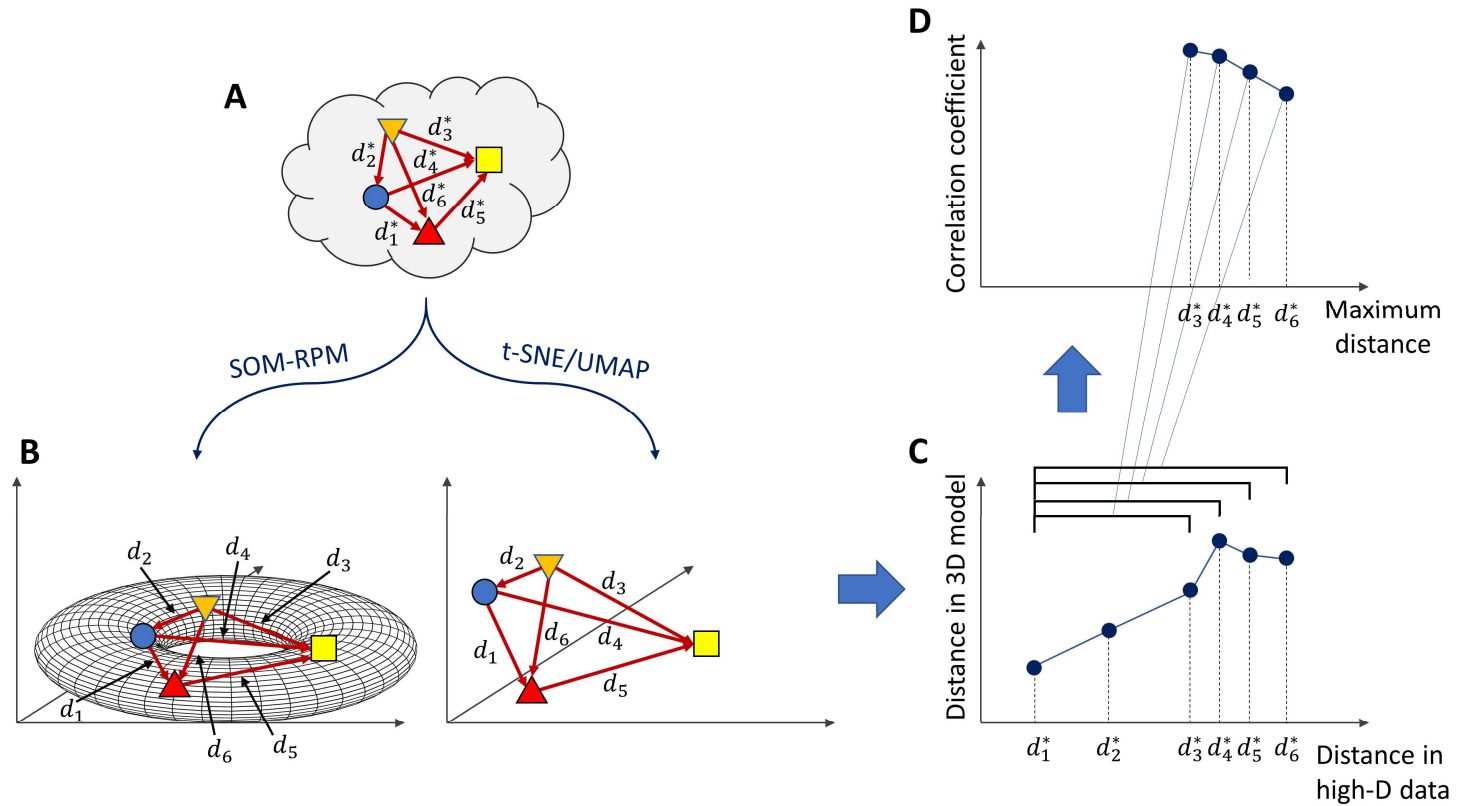

Figure S-1. Fig R1. Schematic showing how the distance correlation metric is calculated, which is used to determine the accuracy of the SOM-RPM, t-SNE and UMAP models with regards to the preservation of distance information. (A) Four points in high-dimensional data space and their associated six distances. (B) The corresponding points in the SOM-RPM (on the surface of a 3D toroid) or t-SNE/UMAP (in the 3D embeddings) models. (C) Plot showing distance in a theoretical 3D model as a function of distance in high-dimensional space. A linear relationship represents a model that accurately preserves distance information. (D) Correlation coefficients for the plot shown in (C), over varying maximum distances. In this example, the model is most accurate up until a distance of $d_{3}^{*}$, beyond which correlation begins to decrease. 

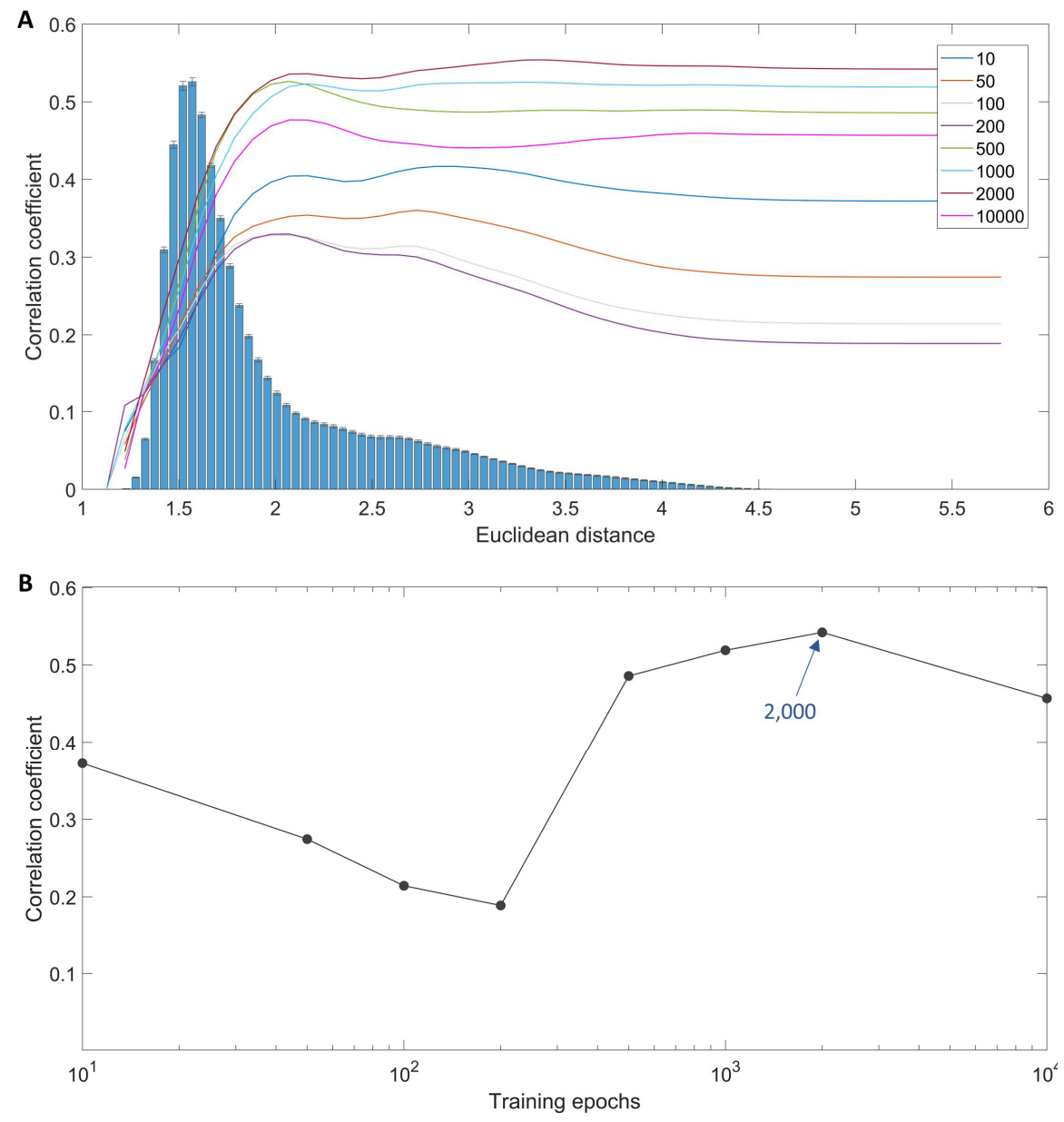

Figure S-2. (A) Plot of Pearson correlation between distance in the SOM-RPM model (using a rigidity of $p=0$ ) and distance in the ToF-SIMS data, over varying maximum distances and for different training epochs (as per the figure legend). Results were used to select the optimum number of training epochs. Plots are overlaid on the pair distribution for the data. (B) Pearson correlation (using the maximum distance in (A)) as a function of training epochs, highlighting the proposed convergence of the SOM-RPM at 2,000 epochs and subsequent overtraining.

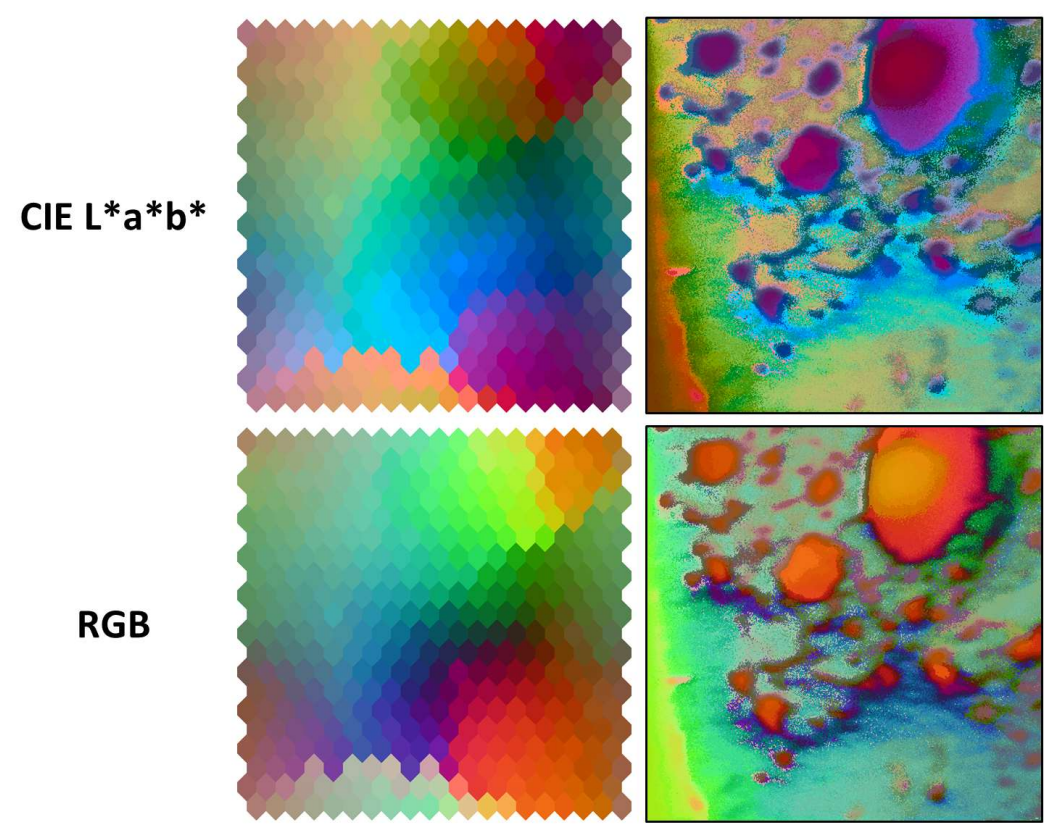


Figure S-3. Comparison of SOM-RPM output using CIE L*a*b* or RGB color schemes, whereby the underlying distance information is identical. Note how the use of color scheme strongly influences how distance information is displayed.
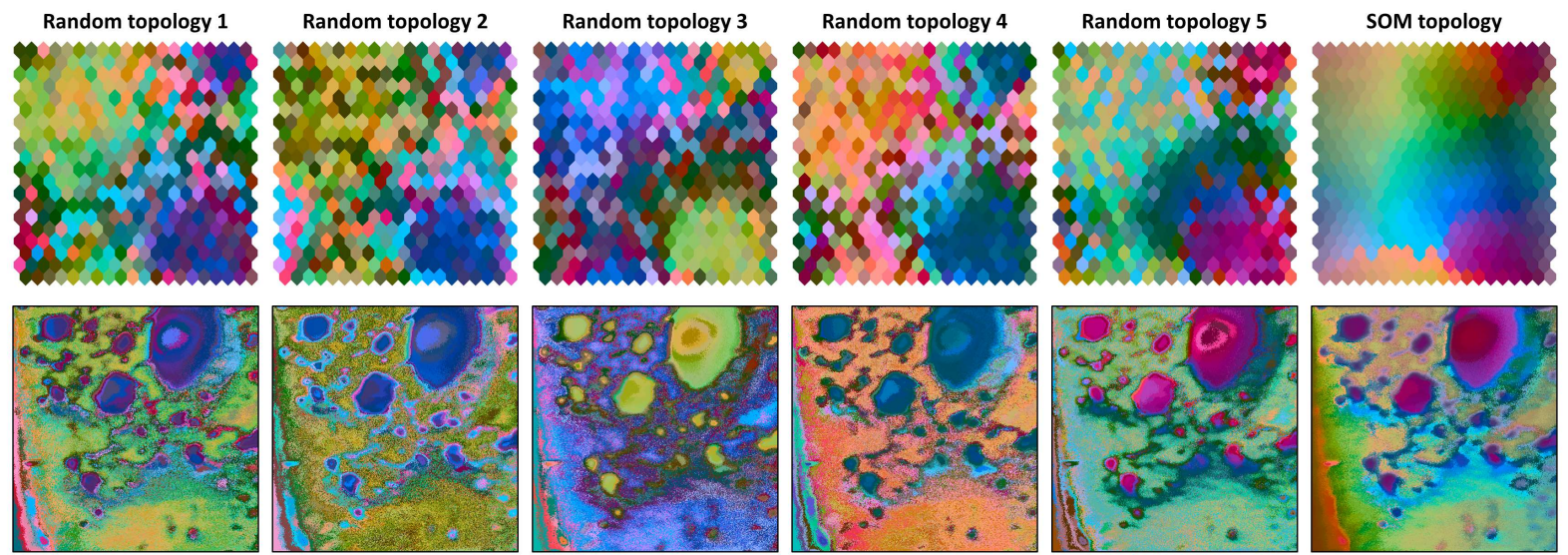

Figure S-4. Colored SOMs (top) and corresponding similarity maps (bottom) produced by the SOM-RPM algorithm, with the topological information randomized $(\boldsymbol{p}=\mathbf{0} ; \boldsymbol{n}=\mathbf{5}$ trials), as well as the same results produced when the topological information provided by the SOM is used.
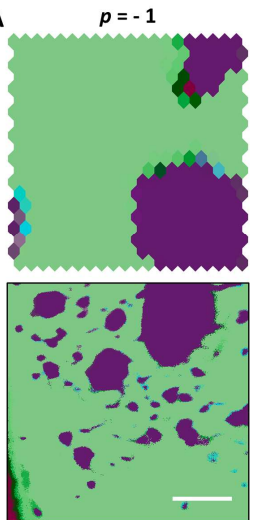

B

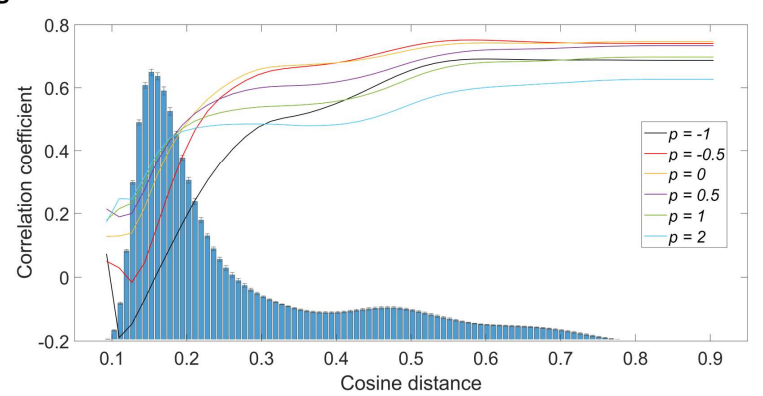

$p=0$
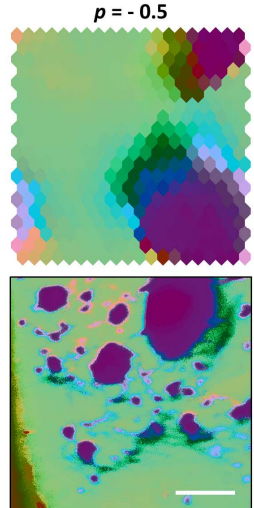
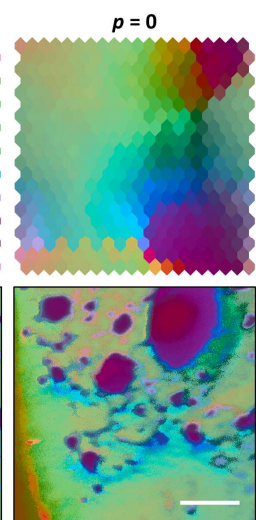

$+$
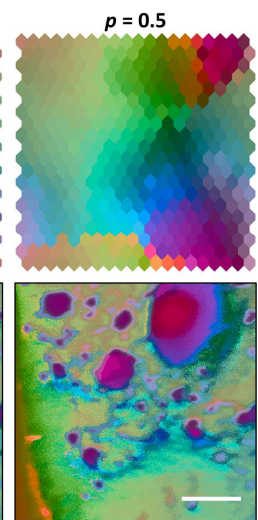
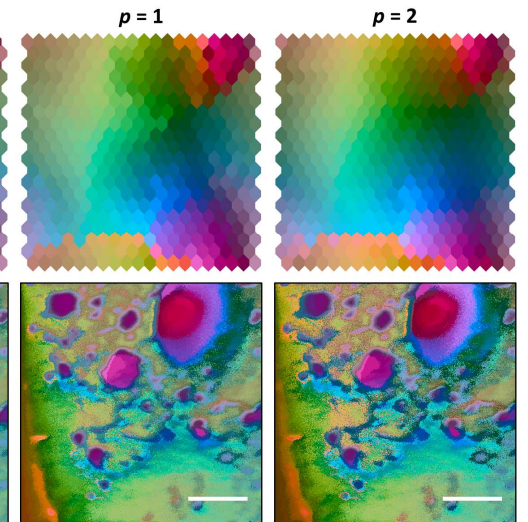

Figure S-5. SOM-RPM analysis of ToF-SIMS imaging data, taken from a mouse breast tumor section. (A) A series of SOMRPM results acquired using different rigidity $(\boldsymbol{p})$ values, with the cosine distance metric. (B) Plot of Pearson correlation between distance in the SOM-RPM model and distance in the ToF-SIMS data, over varying maximum cosine distances and for each rigidity presented in (A). Plots are overlaid on the pair distribution for the data, with cosine distances considered. 


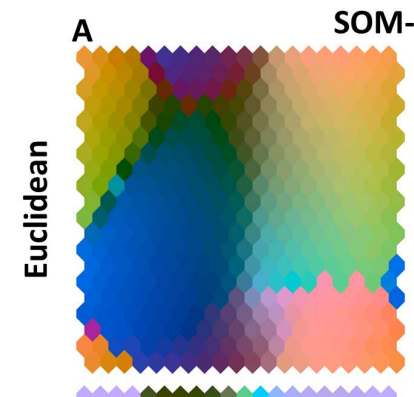

SOM-RPM

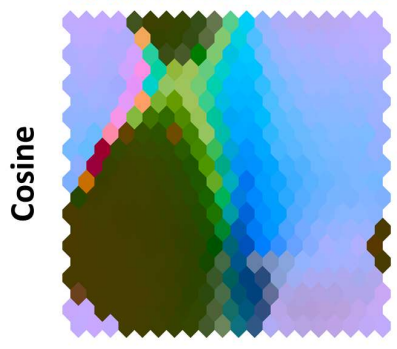

B

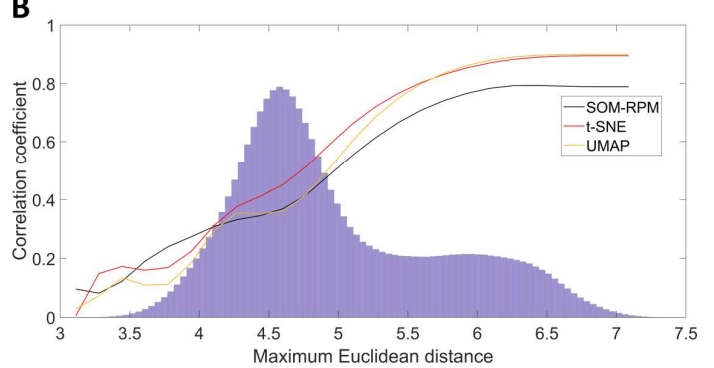

t-SNE
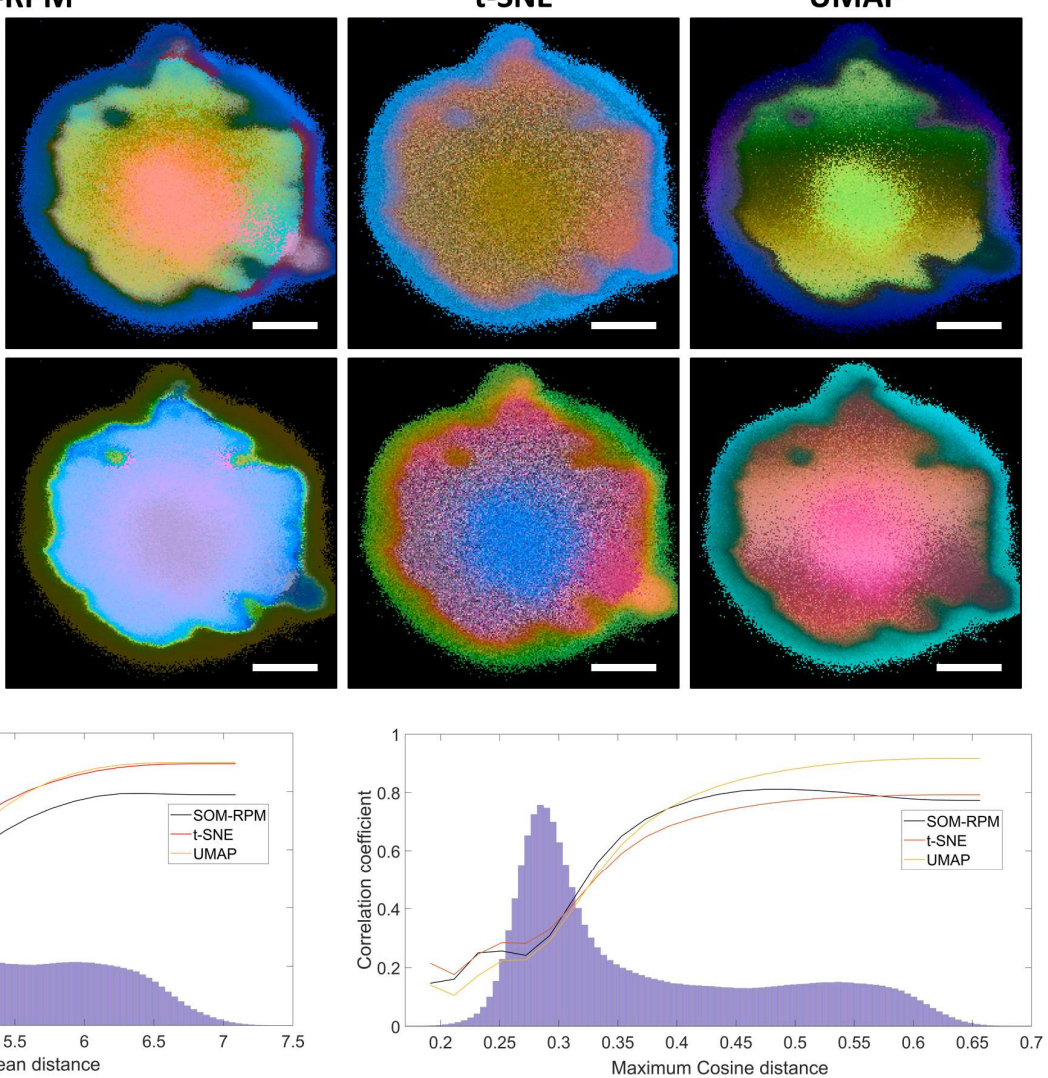

Figure S-6. A comparison between results obtained using SOM-RPM, t-SNE and UMAP algorithms, using both the Euclidean and Cosine distance metrics, for a spheroid cross section. Scale bars are $100 \mu \mathrm{m}$. (A) A comparison of the similarity maps produced. Also shown are the colored SOMs. Scale bars are $100 \mu \mathrm{m}$. (B) Plots of Pearson correlations between model distance and data distance, over varying maximum distances, for each technique and for each distance metric. Plots are overlaid on the pair distributions for the data. For each plot and pair distribution, $10 \%$ of pixels were randomly sampled 5 times, and the means \pm standard deviations are shown. 
1

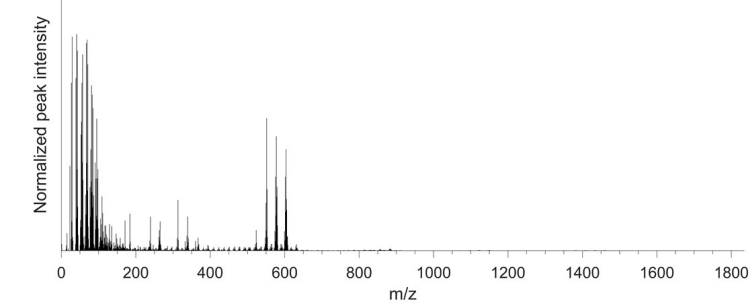

2

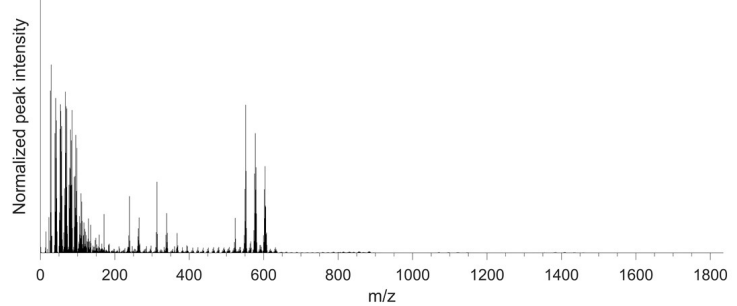

3

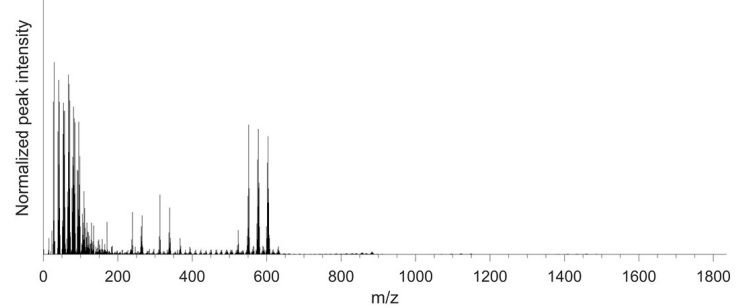

4
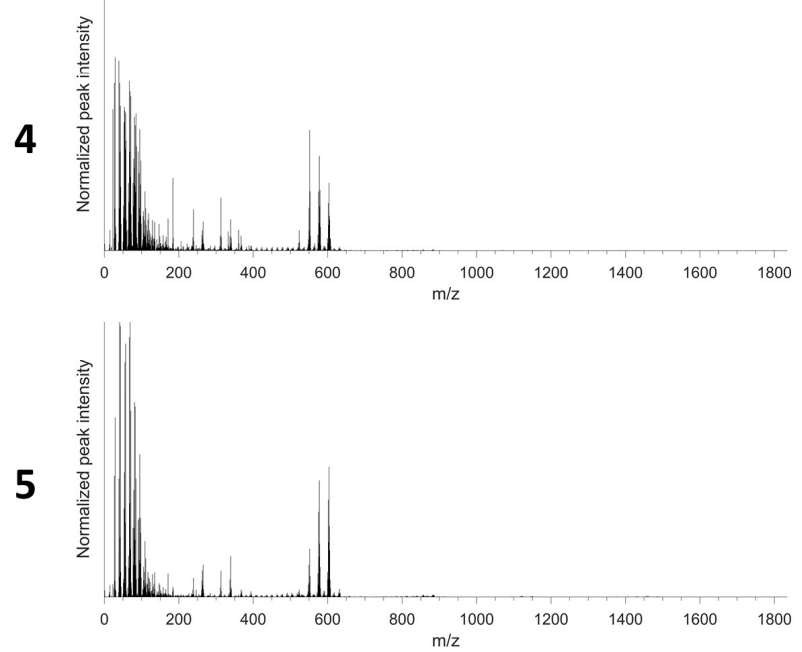
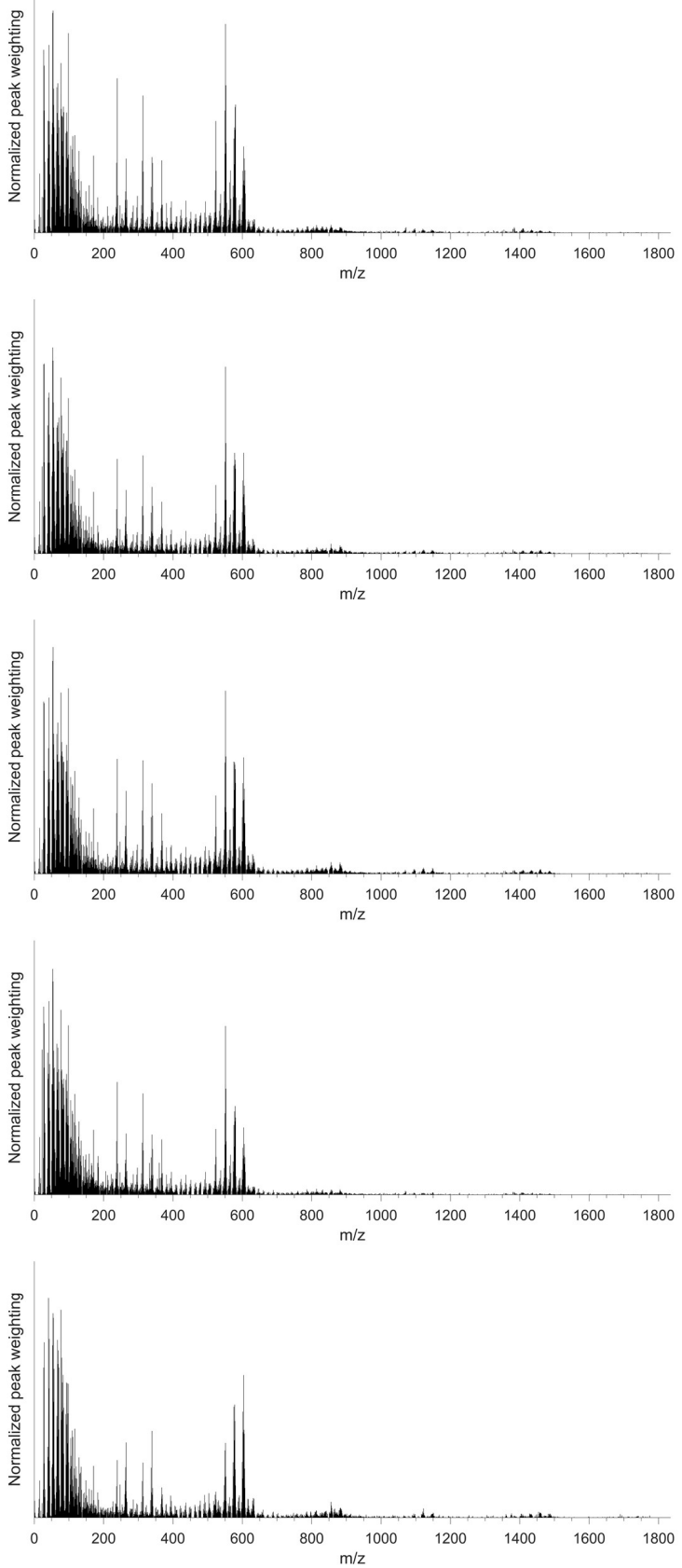

Figure S-7. Mean intensities (left) and weights (right) spectra extracted from each of the five ROIs shown in Fig 6 in the main article text, normalized to total ion count/total weightings for each ROI. 


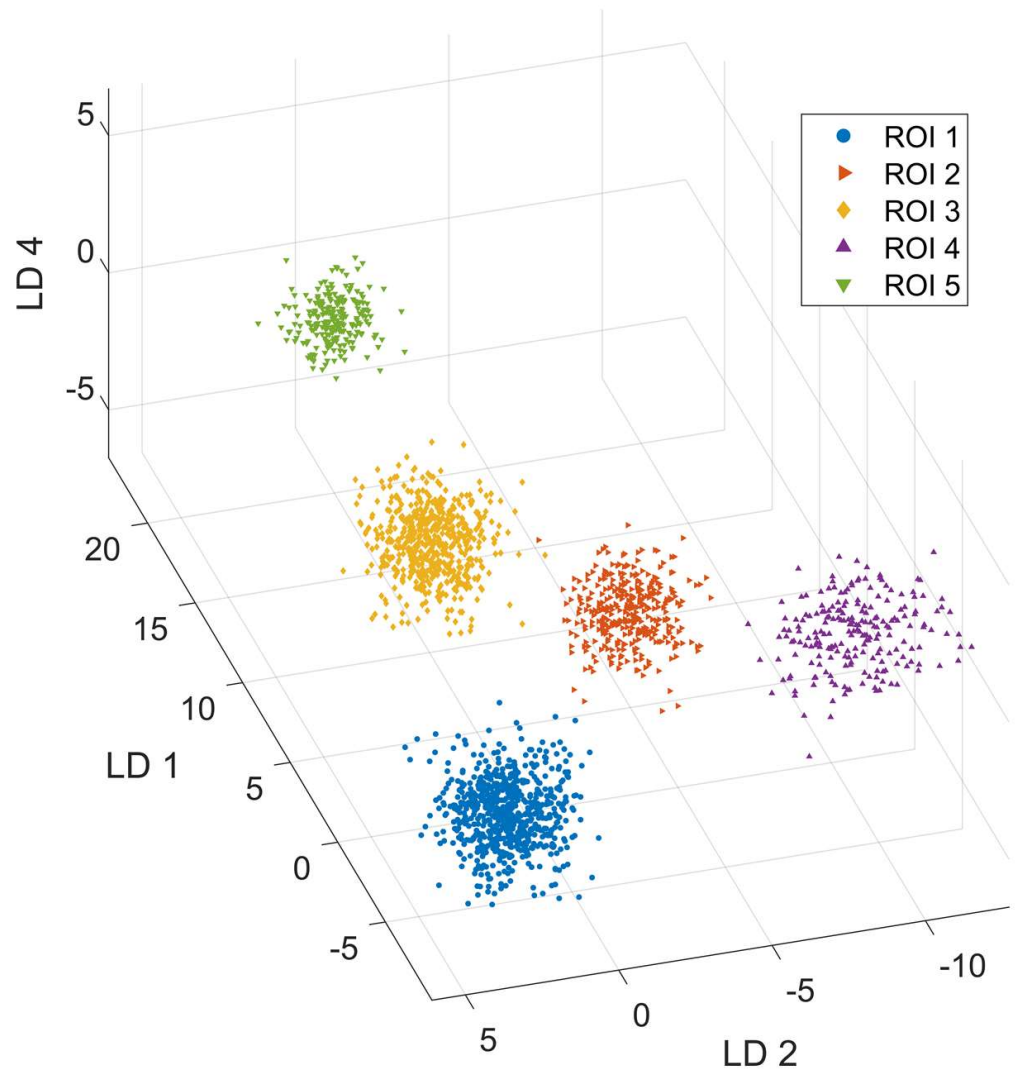

Figure S-8. Results from LDA analysis of the spectra extracted from pixels identified in each ROI in Fig 6 in the main text.

\section{References}

1. Lelekakis, M.; Moseley, J. M.; Martin, T. J.; Hards, D.; Williams, E.; Ho, P.; Lowen, D.; Javni, J.; Miller, F. R.; Slavin, J.; Anderson, R. L., A novel orthotopic model of breast cancer metastasis to bone. Clinical \& Experimental Metastasis 1999, 17 (2), 163-170.

2. $\quad$ Rautela, J.; Baschuk, N.; Slaney, C. Y.; Jayatilleke, K. M.; Xiao, K.; Bidwell, B. N.; Lucas, E. C.;

Hawkins, E. D.; Lock, P.; Wong, C. S.; Chen, W.; Anderson, R. L.; Hertzog, P. J.; Andrews, D. M.; Möller, A.; Parker, B. S., Loss of Host Type-I IFN Signaling Accelerates Metastasis and Impairs NK-cell Antitumor Function in Multiple Models of Breast Cancer. Cancer Immunology Research 2015, 3 (11), 1207-1217.

3. Li, J. X., Visualization of High-Dimensional Data with Relational Perspective Map. Information Visualization 2004, 3 (1), 49-59. 